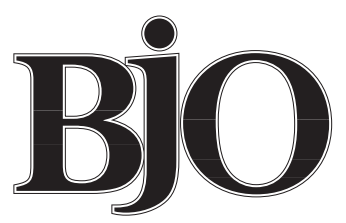

British Journal of Ophthalmology

The 20th century has witnessed great economic, scientific, and technological progress. We have lived in an era of great biotechnological achievements. Our diagnostic and therapeutic abilities in the management of infectious diseases have improved dramatically. Some infections such as smallpox have been eradicated by vaccination, others have been controlled by vigorous public health measures. The resilience of viruses and the tenacity of bacteria have led to the emergence of new infectious diseases and the evolution of old ones. Microbe hunters have won many battles against infections in the 20th century, but the war against classic and emerging infectious diseases is not over. Many new viruses have been discovered over the past two decades including HIV. The degradation of the ecological system, destruction of rain forests, overcrowding and the population boom are exposing humankind to new forms of infectious diseases. With air travel, the world is becoming a true global village and geographic barriers are being disrupted. An infectious agent may jump from the jungles of Africa to the urban communities of the Western hemisphere in a few hours. West Nile virus was recently reported in New York and other eastern states. It is our responsibility to stimulate, encourage, and intensify a continued concerted effort to establish an assault on new and old causes of infectious diseases that may lead to blindness with the ultimate goal of developing new ways to diagnose, new ways to treat, and means to prevent blinding and fatal infections. After the second world war there was a widespread of optimism in the United States that good sanitation, vaccines, and antibiotics would conquer all infectious diseases. This elation and euphoria were of short duration. The control of classic infections was followed by epidemics of infectious diseases. The emergence of new infectious diseases such as AIDS and Ebola virus, and the evolution of old ones such as tuberculosis are becoming serious health problems and important cause of morbidity and mortality. None the less, there has been a dramatic decrease in the incidence of classic infectious diseases over the past four to five decades - a development that can be largely attributed to public health measures, mass vaccination, and the use of new generations of antibiotics.

Trachoma has been recognised since antiquity, and ironically the first effective therapeutic modality using copper sulphate sticks was described by the early Egyptians thousands of years ago, yet trachoma remains a leading cause of blindness in the 21st century in rural Egypt. Currently, the disease afflicts 500 million people and is considered to be among the most common of all human chronic infections, and the most common cause of preventable blindness today. Yet no serious preventive measures or organised campaigns have been conducted in trachoma endemic areas. Active trachoma is seen among people in low socioeconomic groups and appears to be common in underdeveloped and developing countries. It is frequently observed among children who form a large reservoir of the intracellular agent.

With the beginning of the 21 st century, despite Western technology and advances in the field of medicine, trachoma remains a serious health problem leading to ocular morbidity and blindness in many underdeveloped and developing countries, including eastern Mediterranean countries. ${ }^{1}$ In this issue of the BfO, (p 1406) Ezz al Arab and associates have shown that trachoma is a serious health problem in one rural community in Egypt, and remains a major cause of preventable blindness in rural Egypt. Despite the overall socioeconomic development, active trachoma was found among $36.5 \%$ of the children. Severe active trachoma was more common among rural children than urban children. In addition, cicatrising trachoma led to blindness in $8 \%$ of the adult population and accounted for visual impairment in $13 \%$ of the survey members. This means that one out of five individuals above the age of 50 years in one rural community of Egypt suffer from visual loss because of trachoma. This makes trachoma the most important preventable cause of blindness in rural communities of Egypt. This survey underscores the seriousness of trachoma in many developing countries where the health authorities have assumed a less aggressive role in the screening, treatment, and prevention of blindness from trachoma. Medical equipment and pharmaceutical companies have been marketing sophisticated technology and new therapeutic agents to developing nations. In certain country, emphasis on the use of new technology and instruments has been placed ahead of simple treatment of blinding infections such as trachoma that can be treated and controlled by simple inexpensive means. Failure of surgical correction of trichiasis was also noted by Ezz al Arab et al, which points out the importance of continuing medical education and training of health workers for early detection and treatment of trachoma and the management of entropion and trichiasis. Requirements for improving eye care and prevention of blindness from trachoma have been previously outlined. ${ }^{1}$ Other studies have shown the persistence of trachoma as an important cause of blindness in eastern Mediterranean countries. ${ }^{12}$ Prevention of blindness from trachoma may cost less than US $\$ 1$ per patient, 
but the treatment of the complications of the disease such as correction of entropion and trichiasis, dacryocystorhinostomy, and corneal transplantation may cost thousands of dollars. Governmental authorities have ignored insidious non-alarming chronic infections and focused on more conspicuous public health and epidemiological problems such as infant diarrhoea, tuberculosis, and mass vaccination for rubella, diphtheria, measles, and mumps. A realistic approach is to utilise existing primary healthcare centres and resources and to initiate public education and well organised nationwide campaigns for prevention and treatment of trachoma and its complications in rural areas. Continuing medical education programmes for nurses and general practitioners should be established to help in the recognition and treatment of blinding eye diseases.

The clinical and laboratory diagnosis of trachoma is simple. A single oral dose of azithromycin can be safe and effective in the treatment of active trachoma. Surgical correction of entropion and trichiasis is a simple procedure that may lead to the prevention of corneal ulceration, scarring, and blindness. The control of trachoma and its complications can be made available by simple, inexpensive means. This fact should be conveyed to the health authorities in developing countries. The Vision 2020 programme of the World Health Organization (WHO) and the International Agency for the Prevention of Blindness (IAPB) for the elimination of avoidable blindness by the year 2020 will have to place trachoma on the high priority list for the prevention of blindness in developing countries. $^{34}$ Public education for personal hygiene, improving housing conditions, availability of potable water, early detection and treatment of active trachoma, and management of complications of trachoma, such as nasolacrimal duct obstruction, dry eyes, entropion, and trichiasis, can have a major impact on blindness caused by trachoma.

Despite advances in the field of medicine, classic infections such as trachoma remain unconquered major problems in developing countries. Unlike AIDS, trachoma is a curable disease, but control of trachoma is not just a medical problem. Control measures often fail not because of deficiencies in scientific knowledge or public health awareness but because of lack of commitment, lack of resources, political turmoil, and poor socioeconomic policies. In Egypt, as in other developing countries, there have been no recent official governmental programmes for the prevention and treatment of trachoma. Unlike tuberculosis where several strains of Mycobacterium tuberculosis have been identified to be resistant to several antibiotics, new resistant mutants of chlamydia have not been identified. Antimicrobial therapy remains an effective treatment for active trachoma.

Economic development appears to help in decreasing the prevalence and the severity of the disease, but in the study reported by Ezz al Arab and associates, the socioeconomic development did not appear to decrease the prevalence or the severity of trachoma. In a nationwide survey of trachoma in Saudi Arabia, evidence of active or inactive trachoma was found among $22 \%$ of Saudi individuals and $6 \%$ had evidence of active trachoma. ${ }^{2}$ In addition, $17 \%$ of survey members had conjunctival scarring as a result of old trachoma and $1.5 \%$ had entropion and trichiasis. Ten years later, with the socioeconomic development in Saudi Arabia, the prevalence of trachoma decreased to $11 \%$ of the Saudi population, while $2.6 \%$ had active trachoma, suggesting that in one decade there was a dramatic decrease in the prevalence of trachoma and its complications. The prevalence of trachoma in households was directly related in the presence and appearance rating of children in the household, the presence of flies, and the appearance rating of the household itself. ${ }^{2}$ This study showed a decrease in the prevalence of active trachoma in a short period of time in another eastern Mediterranean country, but trachoma prevention and treatment campaigns are urgently needed.

Trachoma is a chronic inflammation that leads to spontaneous resolution accompanied by extensive cicatrisation of the conjunctiva and subconjunctival tissues leading to inward deviation of the eyelashes and lid margins. The trichiasis disrupts the anatomical integrity of the corneal epithelium leading to ulceration and causing opacification of the cornea. In most cases, active trachoma of the conjunctiva may heal without permanent visual loss, but in patients with chronic trachoma the disease may lead to serious damage of the ocular surface. ${ }^{1}$ Obstruction of the lacrimal ductules leads to tear deficiency, and cicatrisation of the conjunctiva leads to loss of goblet cells and mucus deficiency. Similar to trichiasis, dryness of the eye from trachoma may lead to disruption of the anatomical barriers of the corneal epithelium predisposing to secondary infections, corneal scarring, and blindness.

Attempts to prevent trachoma by immunisation with trachoma antigens have been conducted by various groups. The vaccine produced only short immunity to infection. A sensitised subject develops more serious ocular disease than an unimmunised control presumably as a result of the development of hypersensitivity induced by the vaccine. This sensitising effect of trachoma vaccine could be induced in monkeys with low dosage of the antigen and the hypersensitivity reaction is usually more severe than the protective effects of the vaccine. The sensitising effect afforded by the vaccination in contrast to protection was not just strain specific but also type specific. Such results have effectively blocked clinical trials in humans using the whole trachoma agent vaccine. To date there is no effective vaccine for trachoma. Identification of chlamydial antigens that afford protection against the infection without causing a hypersensitivity reaction are highly desirable. Vaccines are getting better and recent technological developments have made it possible to introduce recombinant DNA into mycobacteria such as BCG, the tuberculosis vaccine. It is, therefore, feasible to introduce foreign genes such as DNA or chlamydia and it would be possible to envisage the development of recombinant multiple vaccine vehicles in the future.

The active form of trachoma responds promptly to chemotherapy, but the aftermath of untreated disease will have to be addressed. In children the disease may be treated with topical tetracycline or erythromycin ointment twice daily or systemic erythromycin. Clinical response is slow but the greatest effect occurs in 3-4 months. One of the major problems we face in the treatment of active trachoma is lack of compliance since the majority of cases of active trachoma are asymptomatic. Assigning a 6 week treatment regimen for asymptomatic children may not be practical. Recent studies on azithromycin have shown that the drug is safe in the treatment of active trachoma. Several studies have shown that single dose azithromycin $20 \mathrm{mg} / \mathrm{kg}$ was as effective as topical tetracycline ointment in managing active trachoma. ${ }^{56}$ Single dose azithromycin, therefore, is effective and safe in the treatment of active trachoma; the treatment can establish high compliance and may contribute to the control of trachoma worldwide. In children, azithromycin may be given as a single $20 \mathrm{mg} / \mathrm{kg}$ dose per day and may be repeated in 1 month. Adults may be given 500 $\mathrm{mg}$ of azithromycin orally once daily for 3 days. and the regimen may be repeated in 1 month. The azithromycin levels were assessed in human serum aqueous, tear fluid, and conjunctival tissue specimens after the administration 
of single $1 \mathrm{~g}$ oral dose of azithromycin. ${ }^{7}$ Levels above the $90 \%$ minimal inhibitory concentration $\left(\mathrm{MIC}_{90}\right)$ for Chlamydia trachomatis were found after 4 days in all samples and after 14 days in all conjunctival tissue specimens following oral azithromycin administration. The prolonged high concentration of azithromycin in conjunctival tissue makes this drug suitable for the treatment of conjunctivitis caused by chlamydia.

In conclusion, trachoma is a preventable and curable disease. Prevention of trachoma should be given top priority by the health authorities of countries with endemic trachoma. Mass treatment with azithromycin in schoolchildren such as in $\mathrm{Al}$ Menofiya in Egypt, where one out of three children are infected, may have to be considered. Public health education programmes should be initiated and trachoma control campaigns in countries with endemic trachoma should be started to meet the objective of "Vision 2020" of the WHO and the IAPB.

We cannot rest on the laurels we won for overcoming infections of the past, but we should be prepared for the battle of the 21 st century against classic and emerging infectious diseases.

KHALID F TABBARA

The Eye Center and The Eye Foundation for Research in Ophthalmology, Riyadh, Saudi Arabia

Correspondence to: The Eye Center, PO Box 55307, Riyadh 11534, Saudi Arabia k.tabbara@nesma.net.sa

1 Tabbara KF. Blindness in eastern Mediterranean countries. Br $\mathcal{F}$ Ophthalmol 2001;85:771-5.

2 Tabbara KF, Al-Omar OM. Trachoma in Saudi Arabia. Ophthalmic Epidemiol 1997;4:127-40.

3 Pararajasegaram R. Vision 2020 - the right to sight: from strategies to action. Am f Ophthalmol 1999;128:359-60.

4 Taylor HR, Keefe JE. World blindness: a 21 st century perspective. Br $\mathcal{O} O \mathrm{ph}$ thalmol 2001;85:261-6.

5 Bailey RL, Arullendran P, Whittle HC, et al. Randomized controlled trial of s single-dose azithromycin in treatment of trachom. Lancet 1993:342:453-6.

Tabar $\mathrm{KF}$, Abu El-Asrar AM, Al-Omar O, al Single-dose a:342:453-6. Tabbara KF, Abu El-Asrar AM, Al-Omar O, et al. Single-dose azithromycin in the treatment of trachoma: a randomized, controlled study. Ophthalmol-

Tabbara KF, Al-Kharashi SA, Al-Mansouri SM, et al. Ocular levels of azithromycin. Arch Ophthalmol 1998;116:1625-8.

\section{The role of choroidal haemodynamic abnormalities in the pathogenesis of age related macular degeneration}

Age related macular degeneration (AMD) is the major cause of irreversible blindness in elderly patients worldwide. The pathogenesis of this disease has been investigated from genetic, ${ }^{1}$ histological, ${ }^{2}$ and haemodynamic perspectives. $^{3-6}$ AMD is classified as non-exudative- the dry type, or exudative neovascular-the wet type. ${ }^{78}$ Choroidal neovascularisation (CNV) in the macula causes severe visual impairment in AMD. Several angiogenic factors (vascular endothelial growth factor (VEGF) and basic fibroblast growth factor), which are induced by hypoxia and ischaemia, may play a part in the development of CNV. $^{9-11}$ Choroidal haemodynamics are important for CNV development in AMD.

Chen and colleagues report abnormal choroidal blood flow in asymmetric exudative AMD by measuring pulsatile ocular blood flow (POBF) using an OBF tonometer in 37 patients with asymmetric exudative AMD in this issue of the BFO ( $p$ 1411). Their results demonstrated that POBF was significantly higher in eyes with $\mathrm{CNV}$ than in the contralateral eyes with drusen. Eyes with disciform scars had lower POBF than the contralateral eyes with drusen. There was no significant correlation between the POBF and the size of the CNV lesion. These authors concluded that haemodynamic differences between fellow eyes in individuals are relevant to CNV development and the formation of disciform scars.

We recently studied ocular haemodynamic abnormalities by examining the choroidal blood flow using POBF in patients with AMD. ${ }^{3}$ We used a Langham OBF computerised tonometer in 10 patients with non-exudative AMD, 11 patients with exudative AMD, and 69 age matched control subjects. We reported that POBF and pulse amplitude are lower in the patients with exudative AMD than in the patients with non-exudative AMD and normal subjects. Several angiogenic factors increased in the CNV membranes and vitreous fluid in the patients with AMD. ${ }^{10}{ }^{12}$ In an experimental model of CNV, the expression of VEGF in the accumulating macrophages, migrating retinal pigment epithelial (RPE) cells, and Mueller cells increased. ${ }^{11}$ The RPE cells can promote endothelial proliferation via production and release of VEGF during hypoxia. ${ }^{13}$ These reports suggest that angiogenic factors may play a part in the formation of CNV. In our recent study, POBF in patients with AMD decreased by about $30 \%$ compared with controls and patients with non-exudative AMD. ${ }^{3}$ The decreased total choroidal blood flow may induce $\mathrm{CNV}$ via angiogenic factors resulting from hypoxia in patients with exudative AMD. We concluded that decreased choroidal blood flow may play a part in the development of CNV in AMD.

Several authors investigated choroidal haemodynamic abnormalities in patients with AMD using different blood flow measurements. Ciulla and coworkers reported that the blood velocities of the short posterior ciliary artery are lower in patients with AMD. ${ }^{4}$ Their results indicate that the total choroidal blood flow is lower in patients with AMD compared with controls. Grunwald and coworkers reported that the choroidal blood flow in the centre of the fovea was lower in the patients with non-exudative AMD than in age matched controls, primarily because of decreased blood volume measured by laser Doppler flowmetry. ${ }^{5}$ Friedman and coworkers reported that scleral rigidity increases and choroidal blood flow decreases in patients with $\mathrm{AMD}^{614}{ }^{15}$ and concluded that the eyes of patients with AMD had decreased choroidal blood flow and increased resistance in the choroidal vasculature. There is no doubt that choroidal haemodynamic abnormalities exist in patients with AMD.

Recent treatments, such as laser photocoagulation, photodynamic therapy, and surgery, are efficacious. However, the number of patients who recover good visual acuity after treatment is limited. Thus, there is need for pharmacological therapy that prevents the development of CNV. An understanding of choroidal haemodynamics abnormalities may be important for the development of pharmacological therapy in AMD. For example, we reported that losartan, an angiotensin receptor antagonist, inhibited development of CNV in an experimental model. ${ }^{16}$ Choroidal haemodynamics may have to be assessed to evaluate the inhibitory 
effect of several drugs on CNV development in patients with AMD.

The study of Chen and coworkers showed that POBF was significantly higher in eyes with $\mathrm{CNV}$ than in the contralateral eyes with drusen, and eyes with disciform scars had lower POBF than the contralateral eyes with drusen in exudative AMD. Our recent study published in the $B \mathcal{F O}$ showed that POBF in patients with exudative AMD is lower than in patients with non-exudative AMD and normal subjects. $^{3}$ There seem to be differences in these results using POBF. Chen and coworkers did not conduct a group comparison but an interindividual study in which they evaluated only patients with exudative AMD. In another of our studies, axial length was a major factor affecting POBF in normal subjects. ${ }^{17}$ When evaluating choroidal blood flow by measuring $\mathrm{POBF}$, the effect of axial length should be considered. However, the severity in exudative AMD was not considered in our group comparison study. Therefore, the results of these two studies cannot be compared. Long term follow up and large group comparison studies are needed in the future to gain an understanding of choroidal haemodynamic abnormalities in AMD using POBF and another ocular blood flow measurements.

FUMIHIKO MORI

Department of Ophthalmology, Asahikawa Medical College, Midorigaoka Higashi 2-1-1-1, Asahikawa 078-8510 Japan morinao@d5.dion.ne.jp

1 Allikmets R, Shroyer N, Singh N, et al. Mutation of the Stargardt disease gene (ABCR) in age-related macular degeneration. Science 1997;277:1805-7.
2 Young R. Pathophysiology of age-related macular degeneration. Surv Ophthalmol 1987;31:291-306.

3 Mori F, Konno S, Hikichi T, et al. Pulsatile ocular blood flow study: decreases in exudative age related macular degeneration. Br $\mathcal{F}$ Ophthalmol 2001;85:531-3.

4 Ciulla TA, Harris A, Chung HS, et al. Color Doppler imaging discloses reduced ocular blood flow velocities in nonexudative age-related macular degeneration. Am f Ophthalmol 1999;128:75-80.

5 Grunwald JE, Hariprasad SM, Dupont J, et al. Foveal choroidal blood flow in age-related macular degeneration. Invest Ophthalmol Vis Sci 1998;39: 385-90.

6 Friedman E, Krupsky S, Lane AM, et al. Ocular blood flow velocity in agerelated macular degeneration. Ophthalmology 1995;102:640-6.

7 Sarks SH, Sarks JP. Age-related macular degeneration: atropic form. In: Schachat AP, Murphy RP, eds. Retina. 2nd ed. Vol 2. St Louis: Mosby, 1994:1071-102.

8 Elman MJ, Fine SL. Exudative age-related macular degeneration. In: Schachat AP, Murphy RP, eds. Retina. 2nd ed. Vol 2. St Louis: Mosby, 1994:1103-41.

9 Shweiki D, Itin A, Soffer D, et al. Vascular endothelial growth factor induced by hypoxia may mediate hypoxia-initiated angiogenesis. Nature 1992;359: 843-5.

10 Amin R, Puklin JE, Frank RN. Growth factor localization in choroidal neovascular membranes of age-related macular degeneration. Invest Ophthalmol Vis Sci 1994;35:3178-88.

11 Ishibashi T, Hata Y, Yoshikawa H, et al. Expression of vascular endothelial growth factor in experimental choroidal neovascularization. Graefes Arch Clin Exp Ophthalmol 1997;235:159-67.

12 Wells JA, Murthy R, Chibber R, et al. Levels of vascular endothelial growth factor are elevated in the vitreous of patients with subretinal neovascularisation. Br f Ophthalmol 1996;80:363-6.

13 Adamis AP, Shima DT, Yeo K-T, et al. Synthesis and secretion of vascular permeability factor/vascular endothelial growth factor by human retinal pigment epithelial cells. Biochem Biophys Res Commun 1993;193:631-8.

14 Friedman E, Ivry $M$, Ebert E, et al. Increased scleral rigidity and age-related macular degeneration. Ophthalmology 1989;96:104-8.

15 Friedman E. The role of atherosclerotic process in the pathogenesis of agerelated macular degeneration. Am f Ophthalmol 2000;130: 658-63.

16 Hikichi T, Mori F, Takamiya A, et al. Inhibitory effect of losartan on laserinduced choroidal neovascularization in rats. Am $\mathcal{F}$ Ophthalmol (in press).

17 Mori F, Konno S, Hikichi T, et al. Factors affecting pulsatile ocular blood flow in normal subjects. Br f Ophthalmol 2001;85:529-30.

\section{Amniotic membrane transplantation for persistent corneal epithelial defect}

Persistent corneal epithelial defects with or without ulceration are a serious and urgent clinical problem which can be complicated by microbial infections and thus threaten patients' vision. Experimental studies have revealed that corneal epithelial injuries with or without involvement of the stroma result in rapid healing so long as the eye retains its normal ocular surface defences and possesses healthy limbal epithelial stem cells (for a review see Tseng ${ }^{1}$ ). Therefore, whenever there is a persistent defect or ulcer the diagnostic examination should first be directed to ruling out limbal stem cell deficiency, followed by analysis of the integrity of the ocular surface defence governed by neuroanatomical integration of both trigeminal and facial nerves (for a review see Solomon et $a l^{2}$ ). Although the actual aetiology may arise from multiple influences and be exogenously or endogenously triggered, the most common denominator is neurotrophic keratopathy, in which trigeminal denervation simultaneously abolishes reflexes controlling tear secretion and lid blinking and closure. When medical treatments fail and the defect or ulcer persists (for example, for more than 3 weeks), conventional surgical treatments become indicated and should include punctal occlusion, application of bandage, scleral lens or tissue adhesive, lamellar or full thickness corneal transplantation, tarsorrhaphy, and conjunctival flap.

In this issue of the BFO ( $p$ 1455) Prabhasawat and coworkers reported their clinical experience in using amniotic membrane transplantation for treating 28 patients with persistent corneal epithelial defect or ulcers. As detailed in their report, the majority of eyes (24 of 28,
$86 \%$ ) were indeed affected by neurotrophic keratopathy. Although these authors were not the first reporting the use of amniotic membrane for this indication, theirs is the largest series of seven to date. ${ }^{3-9}$ Consequently, for the first time they could subdivide their patients into three groups according to the severity of stromal thinning, and compare their outcomes by single layer or multilayers of membrane application. They noted an overall success rate of $82.1 \%$, which falls within the reported range of $67 \%$ to $91 \%$. Variable success rates may be attributed to differences in the underlying aetiology and severity, and in the accompanying abnormalities and treatments. Intriguingly, when multilayers were used the defect covered by the amniotic membrane healed significantly faster than that with a single layer. Consistent with what has been reported in seven previous series and two case reports, ${ }^{10}{ }^{11}$ they also noted that ocular surface inflammation is markedly reduced following transplantation (in nearly all cases).

Rapid healing and reduction of ocular surface inflammation following amniotic membrane transplantation can be explained by the following action mechanisms (for details also see references cited in the report by Prabhasawat et al in this issue). Firstly, the amniotic membrane provides a new basement membrane, which is an important substrate for supporting adhesion and growth of epithelial progenitor cells, including stem cells. Secondly, the amniotic membrane exerts an anti-inflammatory effect. We have recently reported that expression of IL $-1 \alpha$ and IL- $1 \beta$ is markedly suppressed when human limbal epithelial cells are cultured on the amniotic membrane stromal matrix, 
even if they are challenged by lipopolysaccharide, a bacterial endotoxin. ${ }^{12}$ Such an anti-inflammatory effect has been demonstrated in experimental injuries caused by excimer laser ablation, ${ }^{13} 14$ acute chemical burns, ${ }^{15}$ or herpes simplex virus type 1 induced necrotising keratitis. ${ }^{16}$ Thirdly, the amniotic membrane stromal matrix has a direct anti-scarring effect as evidenced by its suppression of TGF- $\beta$ signalling and myofibroblast differentiation. Fourthly, the combination of the above three actions may help re-establish a microenvironmental niche that is conducive for the growth of epithelial progenitor cells. Fifthly, the amniotic membrane may promote nerve regeneration by maintaining nerve growth factor (NGF) signalling. Lambiase et $a l^{17}$ first reported that topical application of NGF is effective in healing neurotrophic ulcers. We have recently gathered experimental evidence to confirm that the amniotic membrane contains a large amount of NGF, and preferentially maintains the NGF signalling system for human limbal epithelial cells in culture (Touhami et al, submitted, 2001).

Collectively, the aforementioned mechanisms also explain why the stromal thickness may be restored in deep ulcers using multilayers of membrane, and why some patients regained more vision after transplantation as shown in this and other published reports. However, amniotic membrane transplantation alone cannot heal the persistent defects or ulcers caused by total limbal stem cell deficiency, as noted by Prabhasawat and coworkers and other investigators. Furthermore, amniotic membrane transplantation has a limited role in treating eyes with severe ischaemia (for example, caused by chemical burns or rheumatoid arthritis) and severe exposure (that is, lack of blinking), to which other surgical therapies need to be added. Nevertheless, amniotic membrane transplantation offers the following advantages over conventional corneal transplantation-relative ease of surgery and avoidance of allograft rejection. As a result, amniotic membrane transplantation becomes an attractive alternative for countries where there is a shortage of corneal tissues. Even if corneal transplantation is needed, it can be performed after amniotic membrane transplantation when the ocular surface is not inflamed.
Proprietary interest: Dr Tseng has filed a patent application for the method and uses of amniotic membrane, and has a financial interest in Bio-Tissue, which is a tissue bank providing amniotic membrane for research and clinical uses.

SCHEFFER C G TSENG

Ocular Surface and Tear Center, Department of Ophthalmology, Bascom Palmer Eye Institute and Department of Cell Biology and Anatomy, University of Miami School of Medicine, Miami, FL 33101, USA

Correspondence to: Ocular Surface and Tear Center, Bascom Palmer Eye Institute, William L McKnight Vision Research Pavilion, 1638 NW 10th Avenue, Miami, FL 33136, USA.

stseng@bpei.med.miami.edu

1 Tseng SCG. Regulation and clinical implications of corneal epithelial stem cells. Mol Biol Rep 1996;23:47-58.

2 Solomon A, Touhami A, Sandoval H, et al. Neurotrophic keratopathy: basic concepts and therapeutic strategies. Comp Ophthalmol Update 2000;3:16574

3 Lee S-H, Tseng SCG. Amniotic membrane transplantation for persistent epithelial defects with ulceration. Am f Ophthalmol 1997;123:303-12.

4 Kruse FE, Rohrschneider K, Völcker HE. Multilayer amniotic membrane transplantation for reconstruction of deep corneal ulcers. Ophthalmology $1999 \cdot 106: 1504-11$

5 Azuara-Blanco A, Pillai CT, Dua HS. Amniotic membrane transplantation for ocular surface reconstruction. Br f Ophthalmol 1999;8339:399-402.

6 Chen H-J, Pires RTF, Tseng SCG. Amniotic membrane transplantation for severe neurotrophic corneal ulcers. Br F Ophthalmol 2000;84:826-33.

7 Gabric N, Mravicic I, Dekaris I, et al. Human amniotic membrane in the reconstruction of the ocular surface. Doc Ophthalmol 1999;98:273-83.

8 Hanada K, Shimazaki J, Shimmura S, et al. Multilayered amniotic membrane transplantation for severe ulceration of the cornea and sclera. Am $\mathcal{F}$ Ophthalmol 2001;131:324-31.

9 Letko E, Stechschulte SU, Kenyon KR, et al. Amniotic membrane inlay and overlay grafting for corneal epithelial defects and stromal ulcers. Arch Ophthalmol 2001;119:659-63.

$10 \mathrm{Su}$ CY, Lin CP. Combined use of an amniotic membrane and tissue adhesive in treating corneal perforation: a case report. Ophthalmic Surg Lasers 2000;31:151-4.

11 Peris-Martinez C, Menezo JL, Diaz-Llopis M, et al. Multilayer amniotic membrane transplantation in severe ocular graft versus host disease. Eur $\mathcal{F}$ Ophthalmol 2001;11:183-6.

12 Solomon A, Rosenblatt M, Monroy DC, et al. Suppression of interleukin-1 $\alpha$ and interleukin- $1 \beta$ in the human corneal epithelial cells cultured on the and interleukin-1 $\beta$ in the human corneal epithelial cells cultur

13 Park WC, Tseng SCG. Modulation of acute inflammation and keratocyte death by suturing, blood and amniotic membrane in PRK. Invest Ophthalmol Vis Sci 2000;41:2906-14

14 Wang MX, Gray TB, Parks WC, et al. Corneal haze and apoptosis is reduced by amniotic membrane matrix in excimer laser photoablation in rabbits. $\mathcal{F}$ Cataract Refract Surg 2001;27:310-9.

$15 \mathrm{Kim}$ JS, Kim JC, Na BK, et al. Amniotic membrane patching promotes healing and inhibits protease activity on wound healing following acute corneal alkali burns. Exp Eye Res 1998;70:329-37.

16 Heiligenhaus A, Meller D, Meller D, et al. Improvement of HSV-1 necrotizing keratitis with amniotic membrane transplantation. Invest Ophthalmol Vis Sci 2001;42:1969-74

17 Lambiase A, Rama P, Bonini S, et al. Topical treatment with nerve growth factor for corneal neurotrophic ulcers. $N$ Engl f Med 1998;338:1174-80. 